\title{
Evaluating frequency proximity in stream segregation
}

\author{
KEVIN L. BAKER \\ De Montfort University, Leicester, England \\ SHEILA M. WILLIAMS \\ University of Texas, Austin, Texas \\ and \\ RODERICK I. NICOLSON \\ University of Sheffield, Sheffield, England
}

\begin{abstract}
Consecutive sounds of similar structure that are close in frequency or pitch are more likely to be perceived as part of the same sequence than those at greater frequency separations. The principle of grouping into such perceptual sequences, or auditory streams, is known as frequency proximity. However, the metric by which one frequency difference is judged to be greater or less than another in complex auditory scenes is not yet known. Two experiments explored the metric for frequency proximity. We presented repeating three-tone stimulus patterns at a rate where they are normally heard as two streams, one containing the highest tone and one containing the lowest. The middle tone joined one stream or the other depending on its frequency. Subjects reported the perceived allocation of the variable tone by responding on a 5-point scale. The frequency at which either of these two percepts was equally probable was found to be lower than a logarithmic midpoint or the midpoints on a cochlear map or the Mel scale; that is, it was unlike metrics arrived at by direct comparisons of tones. Further, the midpoint for high and low tones presented synchronously was lower than that for the same tones presented sequentially, demonstrating that in addition to a proximity factor, some additional factor or factors must operate differently when the lower and upper fixed tones are, or are not, presented simultaneously.
\end{abstract}

The segregation of consecutive tones into multiple streams is known to be affected by frequency proximity. Broadly speaking, tones tend to group with others near in frequency and segregate from tones further away. Many composers have exploited this to give the illusion of multiple voices from a single instrument (Bregman, 1990). The musical octave provides a yardstick against which to measure frequency distance so that the proximity of a tone can be quantified. However, outside of the musical context, little is known about the evaluation of frequency proximity in complex sound patterns.

In an early experiment, Miller and Heise (1950) estimated the minimum frequency difference-the trill threshold - for which alternating sequential sinusoids would separate into two distinct streams. Listeners were asked to

This research was carried out at the University of Sheffield, supported by the U.K. MRC/ESRC/SERC Joint Councils Initiative in Cognitive Science (Grant SPG8921799; Green, Williams, and Nicolson). We thank Al Bregman, David Huron, and Quentin Summerfield for their advice on an earlier draft of this paper. We are also grateful for the support and valuable improvements suggested by Bruce Schneider, Steve Greenberg, Valter Ciocca, and especially Donald Greenwood. Correspondence should be addressed to K. L. Baker, Department of Human Communication, De Montfort University, Scraptoft Campus, Leicester LE7 9SU, England (e-mail: klb@)dmu.ac.uk). adjust the frequency of one tone above and one tone below that of a fixed tone for 10 frequencies between 100 and $7000 \mathrm{~Hz}$. They found that for frequencies between $150 \mathrm{~Hz}$ and $1 \mathrm{kHz}$, stream segregation occurred for a frequency difference of $15 \%$. Effective frequency proximity for twotone sequences is thus relatively small and fairly constant throughout this range. This ratio gradually decreased for frequencies between 2 and $7 \mathrm{kHz}$, but the data are not reliable because the response of the earphones used was not perfectly uniform.

Van Noorden (1975) suggested that the streaming phenomenon might be partially explained in terms of the physiology of the inner ear. He proposed that tones presented very rapidly must stimulate the same area of the basilar membrane in order to be integrated into a single coherent stream. His experimental results suggested that, for tones presented at a rate of 10 per second, the largest frequency interval where the observer can still hear the tones as a single stream is around 3 semitones. He further suggested that the frequency interval of this "temporal coherence boundary" increased with longer tone intervals. He hypothesized that pitch motion detectors may exist having only a limited capacity to track rapid changes in frequency, so that with short interonset intervals the mechanism is unable to sustain attention on the stimuli as a single sequence.

In contrast, Bregman $(1978,1990)$ explained stream formation as an accomplishment of "auditory scene anal- 
ysis" and not as the breakdown of a filter-based tracking mechanism. He described the formation of streams as a competition between various pieces of evidence found in the auditory scene. Frequency proximity is one of many primitive grouping processes that participate in this competition. The components of an auditory scene that are close in frequency (or pitch) are considered as likely candidates for a perceptual representation (or stream) of the same acoustic event. However, it is not known whether the evaluation of frequency distance for the components in a stream is related to the perception of pitch distances in psychophysical experiments.

Techniques using masking noise and probe tones have enabled quite precise perceptual and physiological measurement of hearing acuity (Greenwood, 1991; Scharf, 1975). For example, following from earlier work (e.g., Fletcher, 1940; Zwicker, Flottorp, \& Stevens, 1957), Greenwood (1961) formulated a critical band function that is closely proportional to a wide range of critical band data and to equivalent rectangular bandwidth (ERB) data (Greenwood, 1990, 1991), and that may reflect equal cochlear distances on the basilar membrane. A function of the same mathematical form as Greenwood's (somewhat altered constants) has been adopted by Glasberg and Moore (1990) to interpret ERB bandwidths as cochlear distances. Using such information, it is possible to reinterpret the Miller and Heise (1950) data as being more consistent with a theory of critical bandwidths (Plomp \& Steeneken, 1968; Zwicker, 1970), given that critical bands give a higher correlation with the trill threshold than do strictly logarithmic measurements (i.e., equal frequency ratios). Similarly, Greenwood $(1961,1991)$ had found that consonant intervals (see, e.g., Mayer, 1894; Plomp \& Steeneken, 1968; among others) correlated better with critical bands (and equal cochlear distances) than with frequency ratios. Hence a possible candidate for frequency distance could be the frequency-place function (Greenwood, 1961, 1990) whose derivative is closely proportional to critical band and ERB data alike, using proportionality factors suitable for the various bodies of data.

Direct physiological experiments have also been conducted to investigate the response of the basilar membrane to pure tones. Greenwood (1990) summarized the work on frequency versus place of vibration along the basilar membrane and showed that the derivative of the frequency-position function for humans (i.e., his critical bandwidth function of 1961) was very closely proportional to ERB measurements (Houtgast, 1977; Patterson, 1976; Shailer \& Moore, 1983; Weber, 1977 [collated by Moore \& Glasberg, 1983]) and was an excellent description of those data. Greenwood (1991) showed further that the Greenwood (1961) critical band function (the frequency-position function's derivative) was closely proportional to additional ERB data (Moore, Peters, \& Glasberg, 1990; Shailer, Moore, Glasberg, Watson, \& Harris, 1990), to consonant interval data, and (above $400 \mathrm{~Hz}$ ) to the classical critical band data as well, thus showing that each fitted set of data corresponds well to equal distances. All of these studies have used two ton sequences to determine their metrics. Whether the same metrics apply in more dynamic and complex situations, such as the grouping of a tone to one of two possible streams, has not yet been quantified.

Using a variation of Bregman and Pinker's (1978) repeated pattern of three tones, we devised a way of manipulating stream formation on the basis of frequency proximity. The upper tone, $\mathrm{H}$, and the lower tone, L (Figure 1), presented at the same fixed frequencies throughout the experiments, compete with each other to form a stream with a variable third tone, $\mathrm{V}$. The frequency of $\mathrm{V}$ for each trial is selected from a range delimited by the frequencies of $\mathrm{H}$ and $\mathrm{L}$. The tones that are perceptually nearest in frequency will form an alternating tone stream, leaving the furthest tone to form a stream on its own. Asking subjects to describe the perceptual streams formed for different frequencies of $\mathrm{V}$ should allow the frequency proximity metric used in simple streaming situations to be inferred.

It is possible to describe each of the formations by referring to both the tempo and the pitch of the perceptual streams relative to each other. For example, "the slowest stream is higher in pitch."

The competitive nature of stream formation may mean that the effects of frequency proximity appear to vary across different auditory scenes. For this reason we devised two different stimulus patterns: a sequential pattern and a synchronous pattern (Figure 1). The sequential pattern consisted of three discrete tones spaced at equal interonset intervals. This means that the temporal distance from the variable tone to the following high tone is different from that to the following low tone. This forms an auditory scene in which the temporal organization competes with frequency proximity. The synchronous pattern alternated the variable tone with synchronous high and low tones. Here the temporal distance to each potential streaming target is always equal, but the synchrony of the high and low tones may introduce other factors into the competition. To minimize this possibility, the values chosen for the high and low tones were selected to avoid harmonicity and upward or downward masking.

For both patterns, when the frequency of the variable tone, $\mathrm{V}$, is below the perceptual midpoint of the range, $\mathrm{V}$ will tend to group with $L$ to form a stream. Conversely, when it is above the midpoint, it will tend to group with $\mathrm{H}$. When a midpoint has been determined in this way, it will be possible to evaluate whether or not it is also a midpoint on existing scales.

\section{EXPERIMENT 1 The Pitch Task}

\section{Method}

Subjects. Twenty-one subjects were recruited through advertising within the university and were paid for their participation. The age range was 14 to 45 years, although most were under 25 . All reported normal hearing.

Apparatus. All stimuli were prepared and presented diotically at 20,000 samples per second using a Viglen 386 PC computer, run- 

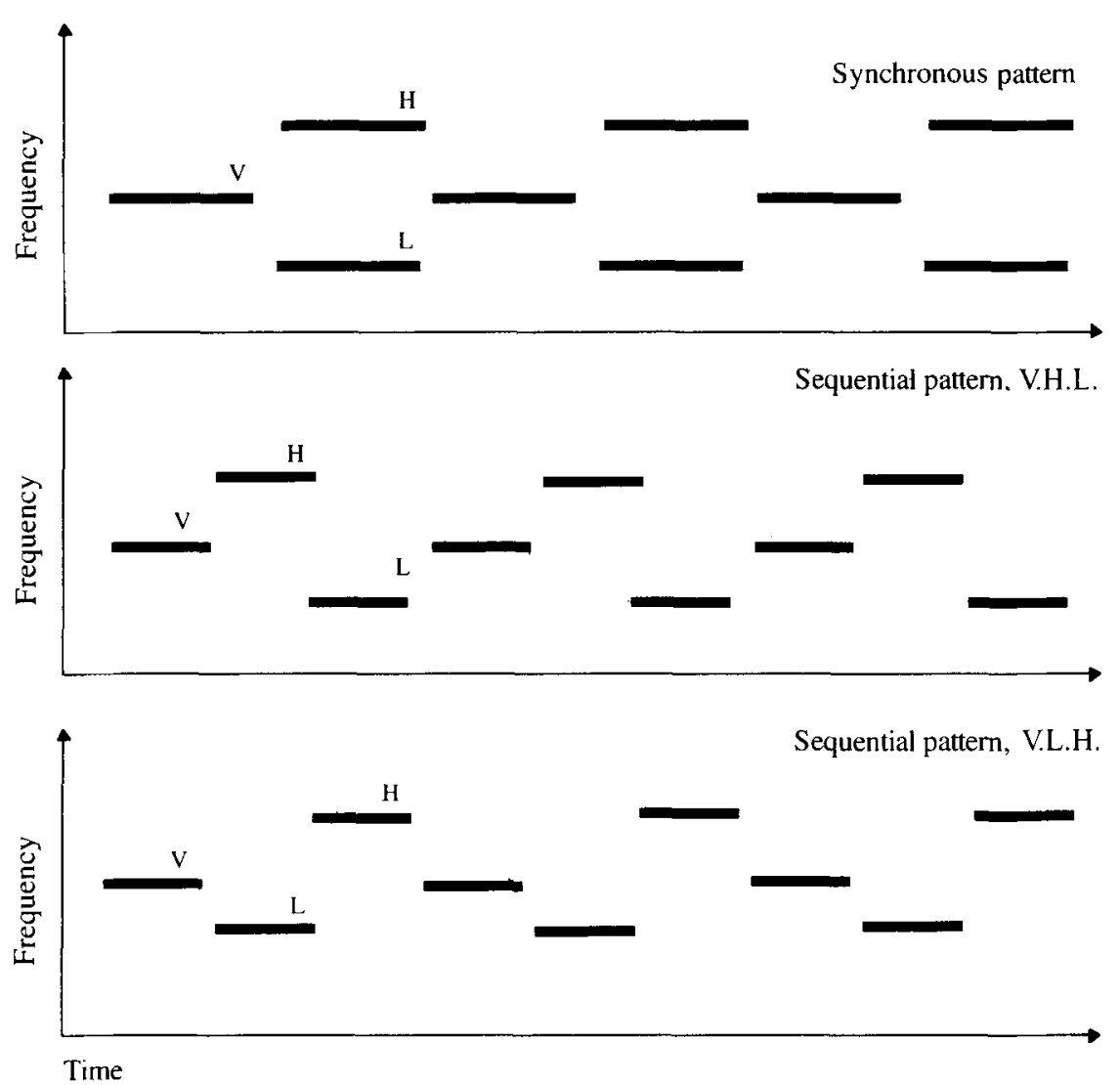

Figure 1. Schematic diagram of the tonal patterns used in the experiments.

ning the MITSYN Version 8.1 (Henke, 1990) sound synthesis system. Each pattern of tones was stored as a signal file and presented as a continuous sequence of 30 repetitions of the pattern. The signals were converted using a Data Translation DT2823 16-bit digitalto-analog board and passed through an $8-\mathrm{kHz}$ cutoff TTE passive filter. The filtered signal was then attenuated using Advance type A64 stepped passive attenuators so that a $1-\mathrm{kHz}$ sinusoid tone was presented at a level of $65 \mathrm{~dB}$ SPL measured at a distance of $1.5 \mathrm{~cm}$ from the headphone coupling. Subjects listened to the sounds through a pair of Beyer Dynamic DT48 headphones while seated in an Industrial Acoustics Co. double-walled acoustic chamber. A program written in MITSYN Command Language (Henke, 1990) was developed to present the stimuli in a random sequence and to collect and record the responses from the subjects' keypresses.

Stimuli. The upper tone, H, and lower tone, L, were set at 1973 and $929 \mathrm{~Hz}$, respectively, covering a relatively flat range of the 65 phon equal loudness contour. In addition to the log and linear midpoints, a range of frequency values was chosen from prime numbers, not within $5 \%$ of any whole-number multiple of any other tone present, to avoid the possibility of simple harmonic relationships between any pair of consecutive tones. The frequency values for the variable tone, V (in Hertz), were 929, 997, 1069, 1129, 1213, 1283 , 1354 (logarithmic midpoint), 1401, 1451 (linear midpoint), 1531, $1627,1709,1801,1889$, and 1973 .

For the synchronous pattern, each tone consisted of a 116-msec steady-state portion, with additional 12-msec linear ramp rise and fall times. There was a $70-$ msec gap between $V$ and the HL complex, and a further $70 \mathrm{msec}$ before the pattern was repeated. The sequential pattern used a steady-state of 86 msec (with the same 12msec ramps) and gaps of $10 \mathrm{msec}$. We found this gave a streaming pattern that was subjectively similar to that of the synchronous pattern. Thus, for both patterns a single repetition of the tonal pattern took $350 \mathrm{msec}$. To balance any ordering effects of the sequential pattern, equal numbers of trials were presented in a rising order, LVH, and a falling order, HVL.

Procedure. Subjects were told that the aim of the experiment was to explore the perceptual grouping of tones. The concept of streaming was explained with the use of analogies to natural acoustic events such as hearing one voice in a crowd and the recognition of vehicle type in traffic noise. This was followed by playing an example of stream organization from a selection of short sequences of four tones. The tones, two high in frequency and two low in frequency, were presented by a computer program at different speeds and presented with written descriptions of what streaming might sound like. This was followed by a short pretest that could be repeated if desired by the listener. The pretest consisted of the tonal patterns with the $\mathrm{V}$ tone set at the extremes of $\mathrm{H}$ and $\mathrm{L}$. The subject was presented twice with each of the patterns in a random order and was asked to describe the streaming using a 5-point scale described below in the Task section. Immediate feedback was given indicating whether the subject was listening to a pattern where $V$ was close in pitch to $\mathrm{H}$, or whether $\mathrm{V}$ was close to $\mathrm{L}$. Subjects who found it difficult to understand the task were excluded from the study at this stage. Once it was established that the subject understood the nature of streaming, the specific experimental instructions were explained.

Sequential and synchronous patterns were presented in separate sessions, on different days, with half the subjects given the sequential pattern first and the others given the synchronous pattern. Subjects were not informed which pattern they were listening to. Each of the 15 stimulus patterns ( 1 for each value of the variable tone) 
was presented six times. For the sequential pattern, equal numbers of each stimulus pattern order were presented. The 90 trials were presented in a different random order for each subject. There was a break of 3 min halfway into each session in addition to optional breaks at a quarter and at three quarters of the way through the session. Including the introductory procedure and explanation, each session took under $50 \mathrm{~min}$ to complete.

Task. Each trial consisted of presenting first $\mathrm{H}$ and then $\mathrm{L}$, separated by a silence of $1 \mathrm{sec}$ as standards, a further silence of $1 \mathrm{sec}$, and then 30 repetitions of the particular stimulus pattern. Throughout the presentation of each trial, the judgment scale and the question, "What is the pitch of the slowest beating stream?" and the reminders " 1 for low" and "5 for high" were presented on the computer display screen. The subject responded after the 30 repetitions of each stimulus pattern using the computer keyboard. We refer to this as the pitch task. The judgment scale ranged from 1 (confident that the slower stream was lowest in pitch) to 5 (confident that the slower stream was highest in pitch). Subjects were asked to respond with a 3 if the percept was changeable or unclear.

\section{Results}

Of the 21 subjects recruited for the experiment, 2 reported that they had misunderstood the task instructions. The random character of their responses reflected this, and consequently their data were excluded from the anal- yses. One subject reported being confused by the stimuli for the synchronous pattern. Given that the data for this subject did not show any consistent pattern of responses even for values of $\mathrm{V}$ at the extremes of the range, these data were also excluded from the analyses. This gave 18 sets of data for the synchronous pattern and 19 for the sequential pattern. Almost all of the subjects commented that the task was easier when they were listening to the sequential pattern. The mean judgments for each position of Tone $V$ for each of the two patterns are shown in Figure 2. The mean judgments are plotted as a function of cochlear distance (from the apex) in addition to the frequency of Tone $V$ since we anticipated the perceptual midpoint to be local to the logarithmic midpoint $(1354 \mathrm{~Hz})$ or a midpoint derived from cochlea distance $(16.102 \mathrm{~mm})$.

Table 1 shows the percentages of all judgments made for each position of Tone $V$ for all subjects. As was expected, the distribution of the judgments was skewed when Tone $\mathrm{V}$ was at the extremes of $\mathrm{H}$ and $\mathrm{L}$.

It was anticipated that a midpoint frequency region would be indicated by a prevalence of " 3 " judgments or a high degree of variability in rating scores. The " 3 " judgments would denote either that the subject was un-

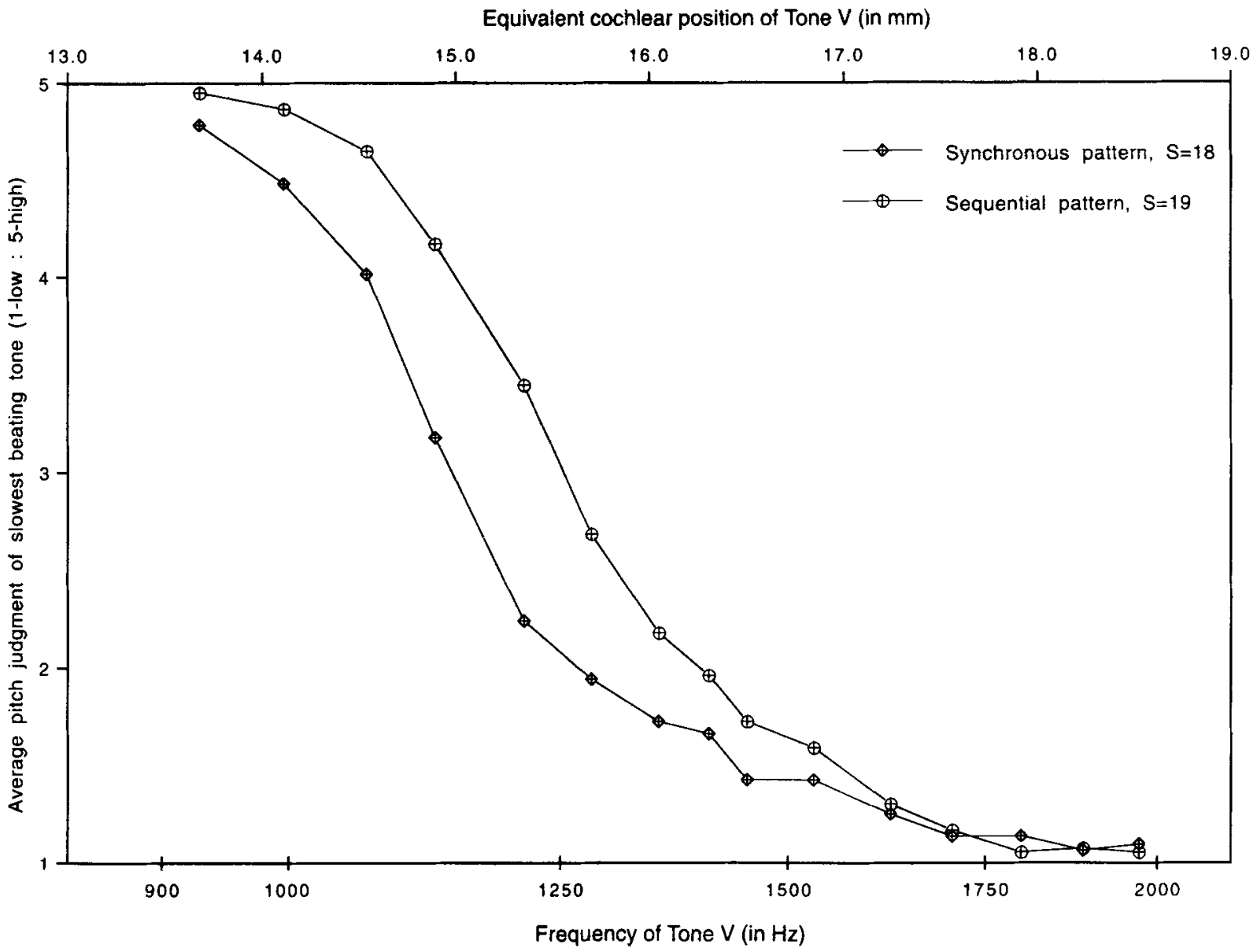

Figure 2. Experiment 1: Average pitch judgments of the slowest beating tone as a function of the frequency (and assumed cochlear position) of Tone V. Cochlear distance was calculated following Greenwood (1991). 
Table 1

Judgments of the Pitch of the Slowest Beating Tone,

Expressed as Percentages of the Total Number of Judgments Made by All Subjects at Each Frequency Position of Tone $\mathrm{V}$

\begin{tabular}{|c|c|c|c|c|c|c|c|c|c|c|c|c|c|c|c|}
\hline \multirow[b]{2}{*}{ Judgment } & \multicolumn{15}{|c|}{ Frequency of Tone $\mathrm{V}$ in Hertz } \\
\hline & 929 & 997 & 1069 & 1129 & 1213 & 1283 & 1354 & 1409 & 1451 & 1531 & 1627 & 1709 & 1801 & 1889 & 1973 \\
\hline \multicolumn{16}{|c|}{ Synchronous Pattern } \\
\hline 1 & 0.9 & 4.6 & 11.1 & 27.8 & 48.1 & 58.3 & 63.0 & 63.9 & 74.1 & 75.9 & 83.3 & 89.8 & 90.7 & 95.4 & 94.4 \\
\hline 2 & 2.8 & 4.6 & 3.7 & 8.3 & 13.0 & 12.0 & 13.9 & 16.7 & 13.9 & 13.0 & 10.2 & 7.4 & 6.5 & 3.7 & 3.7 \\
\hline 3 & 0.9 & 2.8 & 12.0 & 12.0 & 17.6 & 13.9 & 13.9 & 12.0 & 8.3 & 4.6 & 4.6 & 1.9 & 0.9 & 0.0 & 0.9 \\
\hline 4 & 8.3 & 13.9 & 18.5 & 21.3 & 9.3 & 8.3 & 5.6 & 3.7 & 2.8 & 5.6 & 1.9 & 0.9 & 1.9 & 0.9 & 0.0 \\
\hline 5 & 87.0 & 74.1 & 54.6 & 30.6 & 12.0 & 7.4 & 3.7 & 3.7 & 0.9 & 0.9 & 0.0 & 0.0 & 0.0 & 0.0 & 0.9 \\
\hline$M$ & 4.78 & 4.48 & 4.02 & 3.19 & 2.24 & 1.94 & 1.73 & 1.67 & 1.43 & 1.43 & 1.25 & 1.14 & 1.14 & 1.06 & 1.09 \\
\hline$S D$ & & & & 1.6 & 1.4 & 1.31 & 1.12 & 1.06 & 0.83 & 0.88 & 0.63 & 0.46 & 0.50 & 0.34 & 0.46 \\
\hline Skew & -3.71 & -2.22 & -1.23 & -0.27 & 0.75 & 1.13 & 1.43 & 1.64 & 2.11 & 2.23 & 2.75 & 3.95 & 4.34 & 6.77 & 6.61 \\
\hline \multicolumn{16}{|c|}{ Sequential Pattern } \\
\hline 1 & 0.9 & 1.8 & 2.6 & 13.2 & 26.3 & 43.0 & 56.1 & $58.8^{\circ}$ & 67.5 & 74.6 & 83.3 & 89.5 & 94.7 & 93.9 & 96.5 \\
\hline 2 & 0.0 & 0.9 & 2.6 & 3.5 & 7.0 & 10.5 & 12.3 & 14.0 & 12.3 & 9.6 & 7.9 & 6.1 & 4.4 & 4.4 & 2.6 \\
\hline 3 & 0.0 & 0.0 & 6.1 & 4.4 & 10.5 & 12.3 & 7.9 & 11.4 & 8.8 & 5.3 & 5.3 & 3.5 & 0.9 & 1.8 & 0.0 \\
\hline 4 & 2.6 & 4.4 & 5.3 & 11.4 & 6.1 & 3.5 & 4.4 & 3.5 & 2. & 3.5 & 1.8 & 0.0 & 0.0 & 0.0 & 0.0 \\
\hline 5 & 96.5 & 93.0 & 83.3 & 67.5 & 50.0 & 30.7 & 19.3 & 12.3 & 8.8 & 7.0 & 1.8 & 0.9 & 0.0 & 0.0 & 0.9 \\
\hline$M$ & 4.94 & 4.86 & 4.64 & 4.17 & 3.46 & 2.68 & 2.18 & 1.96 & 1.73 & 1.59 & 1.31 & 1.17 & 1.06 & 1.08 & 1.06 \\
\hline$S D$ & & & & 1.42 & 1.72 & 1.73 & 1.59 & 1.40 & 1.26 & 1.18 & 0.80 & 0.56 & 0.27 & 0.33 & 0.40 \\
\hline Skew & -8.59 & -5.28 & -2.70 & -1.49 & -0.47 & 0.36 & 0.93 & 1.24 & 1.68 & 2.01 & 2.98 & 4.27 & 4.91 & 4.53 & 8.59 \\
\hline
\end{tabular}

Note-The means, standard deviations, and skew are derived from the actual judgments. There were 18 sets of data for the synchronous pattern and 19 for the sequential pattern.

clear about the stream formation or that the percept changed over the length of the presentation. Alternatively, if streaming was always clearly established, a mixture of "5" and " $\mathrm{l}$ " judgments would be made when V was near to the midpoint, leading to a nigh variation in the judgments recorded. In both cases a mean judgment would be near to " 3 ."

For the synchronous pattern, the mean judgment nearest to 3 (3.19) and the highest standard deviation (1.61) was when Tone $V$ was at $1129 \mathrm{~Hz}$. The distribution of the streaming judgments seems to be bimodal at this frequency and is possibly a transition between $1069 \mathrm{~Hz}$, where the most frequent judgment is 5 , and $1213 \mathrm{~Hz}$, where the most frequent judgment is 1 . The highest number of " 3 " judgments occurred when $V$ was at $1213 \mathrm{~Hz}$. This suggested a midpoint near to, or between, the frequencies of 1129 and $1213 \mathrm{~Hz}$.

The sequential pattern yielded similar results. The mean judgment nearest to 3 was when $V$ was at $1213 \mathrm{~Hz}$ (3.46), and again showed one of the highest standard deviations (1.72). The highest standard deviation of the judgments (1.73) was when V was at $1283 \mathrm{~Hz}$, which also showed the highest percentage of " 3 " judgments (12.3\%). The distribution of judgments changes from predominately 5 for $1129 \mathrm{~Hz}$ to predominantly 1 for $1354 \mathrm{~Hz}$ either side of these frequencies of 1213 and $1283 \mathrm{~Hz}$, which show near-bimodal distributions. This indicates a possible midpoint between 1213 and $1283 \mathrm{~Hz}$.

In order to make a more accurate estimate of the midpoint, the mean judgments for each frequency value of Tone $\mathrm{V}$ for every subject were analyzed using probit analysis (Finney, 1971), a technique developed for the analy- sis of judgment data expected to follow a sigmoidal pattern. Probit analysis determines the 50\% threshold, which can be interpreted as the perceptual midpoint for the tonal patterns based on the response judgments for all the patterns presented to each subject. The resulting midpoints for each subject were then aggregated to give an average midpoint representative of the subject group. This gave a midpoint for the synchronous pattern at $1179 \mathrm{~Hz}$ $(S D=81.4)$ and for the sequential pattern at $1288 \mathrm{~Hz}$ $(S D=112.2)$. These are considerably below the log midpoint of $1354 \mathrm{~Hz}$ for the range we were studying.

It is possible to treat these aggregated midpoints as sample means and consequently compare them with a population mean of the expected log midpoint (of $1354 \mathrm{~Hz}$ ) using a $t$ distribution. For the synchronous pattern, the observed midpoints differed significantly from the expected midpoint $[t(17)=-9.1347, p<.001]$. Although the midpoint for the sequential pattern was nearer to the expected midpoint, this too was significantly different $[t(18)=-2.5622, p<.01]$.

Discussion of the implications of this result is deferred until after the description of the second experiment.

\section{EXPERIMENT 2 The Tempo Task}

Because streaming has been demonstrated to be attention dependent (van Noorden, 1975), we decided to explore the possibility that focusing on the temporal structure of the resulting streams might have influenced the streaming threshold measured in Experiment 1. Thus Experiment 2 employed a different task. 


\section{Method}

The same stimuli were presented to a different cohort of subjects, who were asked to make a judgment of the tempo of the lowest pitched stream. Essentially, the subjects in both experiments were asked to judge the same streaming phenomena, but focused on different aspects of the stream percept.

Subjects. Twenty-four subjects were recruited in the same way as for Experiment 1. The age range for these subjects was between 17 and 38 years, although most were under 25 . All subjects reported normal hearing.

Apparatus and Stimuli. The apparatus and stimulus patterns were exactly the same as for Experiment 1.

Procedure. The design and procedure were the same as in the previous experiment. Only the instructions to the subjects and the reference tones that preceded the stimuli differed.

Task. Instead of two initial standard tones corresponding to the frequencies of $\mathrm{H}$ and $\mathrm{L}$, a single tone at $929 \mathrm{~Hz}$ (the lower boundary value) was presented for half a second. This was intended to give the subjects a standard by which to identify the lowest pitched stream. This standard tone was followed by $1 \mathrm{sec}$ of silence and then the repetitions of the particular tonal pattern for that trial.

Throughout the presentation of each trial, the judgment scale and the question "What is the speed of the lowest pitched stream?" and the reminders "I for slow" and " 5 for fast" were presented on the computer display screen. The subject responded using the computer keyboard. We refer to this as the tempo task. Again, a judgment of 3 was requested to indicate that the percept was changeable or unclear. The training procedure was the same as for Experiment 1.

\section{Results}

Using the same criteria as for Experiment 1, we had to discard a much larger number of response sets. Additionally, it was found that few subjects could complete the task using the synchronous pattern without extensive practice. For this reason, 15 of the subjects completed the sequential task first. Of the 24 subjects who participated in the experiment, 18 successfully completed the task using the sequential pattern and 17 using the synchronous pattern. The mean judgments for each of the two patterns are shown in Figure 3.

Table 2 summarizes the judgments made for each position of Tone $\mathrm{V}$ for all subjects. The table indicates a similar pattern of judgments to those found for Experiment 1 . For the synchronous pattern, the mean judgment nearest to $3(3.18)$, and the highest standard deviation (1.61) was when Tone $V$ was at $1129 \mathrm{~Hz}$. The distribution of judgments switches from mostly 5 at $1069 \mathrm{~Hz}$ to mostly

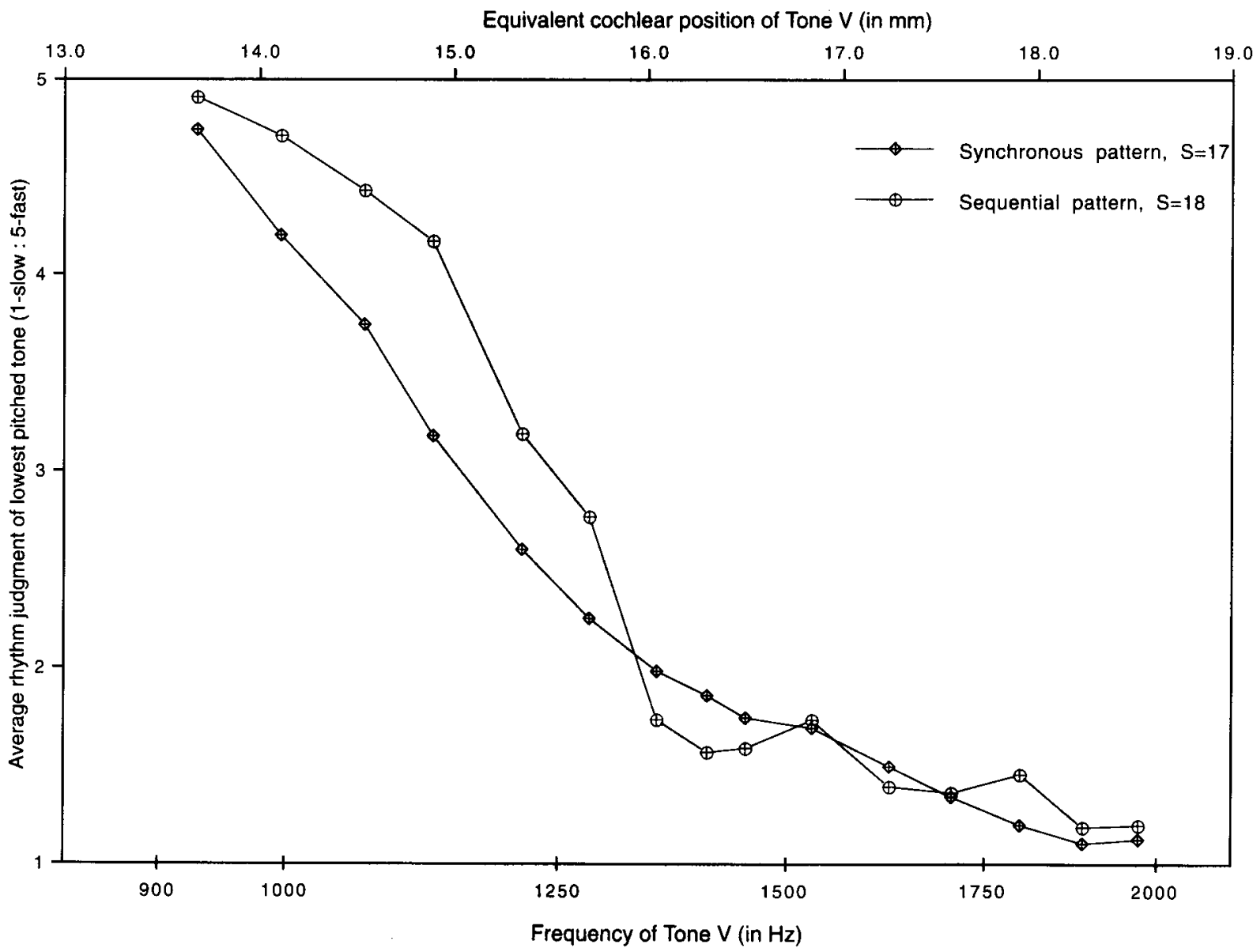

Figure 3. Experiment 2: Average rhythm judgments of the lowest pitched stream as a function of the frequency (and assumed cochlear position) of Tone V. Cochlear distance was calculated following Greenwood (1991). 
Table 2

Judgments of the Rhythm of the Lowest Pitched Stream,

Expressed as Percentages of the Total Number of Judgments Made by All Subjects at Each Frequency Position of Tone $V$

\begin{tabular}{|c|c|c|c|c|c|c|c|c|c|c|c|c|c|c|c|}
\hline \multirow[b]{2}{*}{ Judgment } & \multicolumn{15}{|c|}{ Frequency of Tone $\mathrm{V}$ in Hertz } \\
\hline & 929 & 997 & 1069 & 1129 & 1213 & 1283 & 1354 & 1409 & 1451 & 1531 & 1627 & 1709 & 1801 & 1889 & 1973 \\
\hline \multicolumn{16}{|c|}{ Synchronous Pattern } \\
\hline 1 & 2.9 & 6.9 & 14.7 & 26.5 & 39.2 & 49.0 & 55.9 & 60.8 & 71.6 & 71.6 & 75.5 & 81.4 & 86.3 & 93.1 & 92.2 \\
\hline 2 & 1.0 & 7.8 & 6.9 & 9.8 & 13.7 & 10.8 & 15.7 & 12.7 & 5.9 & 10.8 & 10.8 & 7.8 & 10.8 & 3.9 & 5.9 \\
\hline 3 & 3.9 & 8.8 & 17.6 & 16.7 & 16.7 & 20.6 & 12.7 & 14.7 & 8.8 & 3.9 & 6.9 & 6.9 & 0.0 & 2.0 & 0.0 \\
\hline 4 & 3.9 & 10.8 & 10.8 & 13.7 & 8.8 & 4.9 & 5.9 & 2.9 & 3.9 & 3.9 & 2.0 & 2.9 & 2.0 & 1.0 & 1.0 \\
\hline 5 & 88.2 & 65.7 & 50.0 & 33.3 & 21.6 & 14.7 & 9.8 & 8.8 & 9.8 & 9.8 & 4.9 & 1.0 & 1.0 & 0.0 & 1.0 \\
\hline$M$ & 4.74 & 4.21 & 3.75 & 3.18 & 2.60 & 2.25 & 1.98 & 1.86 & 1.75 & 1.70 & 1.50 & 1.34 & 1.21 & 1.11 & 1.13 \\
\hline$S D$ & 0.83 & 1.28 & 1.49 & 1.61 & 1.58 & 1.47 & 1.34 & 1.28 & 1.33 & 1.30 & 1.05 & 0.81 & 0.63 & 0.44 & 0.54 \\
\hline Skew & -3.47 & -1.42 & -0.77 & -0.19 & 0.42 & 0.78 & 1.17 & 1.36 & 1.59 & 1.77 & 2.27 & 2.54 & 4.07 & 4.70 & 5.52 \\
\hline \multicolumn{16}{|c|}{ Sequential Pattern } \\
\hline 1 & 0.0 & 1.9 & 5.6 & 8.3 & 24.1 & 32.4 & 62.0 & 67.6 & 66.7 & 63.9 & 75.9 & 78.7 & 76.9 & 87.0 & 88.0 \\
\hline 2 & 1.9 & 0.0 & 1.9 & 2.8 & 13.9 & 20.4 & 16.7 & 16.7 & 15.7 & 13.9 & 14.8 & 11.1 & 10.2 & 10.2 & 7.4 \\
\hline 3 & 0.9 & 7.4 & 10.2 & 15.7 & 16.7 & 12.0 & 12.0 & 8.3 & 12.0 & 11.1 & 3.7 & 5.6 & 6.5 & 0.0 & 1.9 \\
\hline 4 & 2.8 & 7.4 & 9.3 & 10.2 & 9.3 & 8.3 & 3.7 & 5.6 & 2.8 & 6.5 & 4.6 & 3.7 & 2.8 & 1.9 & 1.9 \\
\hline 5 & 94.4 & 83.3 & 73.1 & 63.0 & 36.1 & 26.9 & 5.6 & 1.9 & 2.8 & 4.6 & 0.9 & 0.9 & 3.7 & 0.9 & 0.9 \\
\hline$M$ & 4.90 & 4.70 & 4.43 & 4.17 & 3.19 & 2.77 & 1.74 & 1.57 & 1.59 & 1.74 & 1.40 & 1.37 & 1.46 & 1.19 & 1.20 \\
\hline$S D$ & 0.47 & 0.76 & 1.11 & 1.27 & 1.61 & 1.61 & 1.15 & 0.98 & 0.99 & 1.17 & 0.84 & 0.82 & 0.99 & 0.62 & 0.65 \\
\hline Skew & -5.20 & -3.02 & -1.99 & -1.38 & -0.15 & 0.30 & 1.56 & 1.78 & 1.76 & 1.48 & 2.38 & 2.44 & 2.34 & 4.20 & 3.89 \\
\hline
\end{tabular}

Note-The means, standard deviations, and skew are derived from the actual judgments. There were 17 sets of data for the synchronous pattern and 18 for the sequential pattern.

1 at $1283 \mathrm{~Hz}$, suggesting a midpoint between 1129 and $1213 \mathrm{~Hz}$ displaying near-bimodal distribution.

The judgments for the sequential pattern were also similar to those found in Experiment 1. The mean nearest to 3 (3.19) and the highest standard deviation (1.61) was when Tone $\mathrm{V}$ was at $1213 \mathrm{~Hz}$. The distribution of judgments switched from unimodal 5 at $1129 \mathrm{~Hz}$ to bimodal between 1213 and $1283 \mathrm{~Hz}$ to unimodal 1 at $1354 \mathrm{~Hz}$. This suggests a midpoint near to or around $1213-1283 \mathrm{~Hz}$.

Using probit analysis, we obtained an average midpoint for the subject group for the synchronous pattern at $1182 \mathrm{~Hz}(S D=201.5)$ and for the sequential pattern at $1278 \mathrm{~Hz}(S D=79.88)$. These results are again well below the log midpoint for the range and are similar to the results found in Experiment 1 .

We next compared these midpoints with the expected $\log$ midpoint using a $t$ distribution. The midpoints for the synchronous pattern were significantly different from an expected mean midpoint of $1354 \mathrm{~Hz}[t(16)=-3.5020$, $p<.01]$. Again, even though the midpoint for the sequential pattern was nearer to the expected $\log$ midpoint, this too was significantly different from an expected mean midpoint of $1354 \mathrm{~Hz}[t(17)=-4.0367, p<.001]$.

\section{DISCUSSION}

To summarize, we measured the frequency of the perceptual midpoints for the grouping of a target tone with tones of 1973 or $929 \mathrm{~Hz}$ to be $1179 \mathrm{~Hz}$ for the synchronous pattern and $1288 \mathrm{~Hz}$ for the sequential pattern in Experiment 1 , using the pitch task. For the tempo task used in Experiment 2, we measured the frequency of the perceptual midpoints to be $1182 \mathrm{~Hz}$ for the synchronous pattern and $1278 \mathrm{~Hz}$ for the sequential pattern.
The two experiments indicated a midpoint of approximately $1180 \mathrm{~Hz}$ for the synchronous pattern and $1280 \mathrm{~Hz}$ for the sequential pattern. The difference between the subjects' midpoints suggested by the probit analysis for each pattern was statistically significant only for Experiment 1 , using the pitch task $[F(1,35)=8.727, p<.01]$. In Experiment 2, many subjects reported difficulty in making confident judgments of the tempo of the lowest pitched stream. This was reflected in the higher number of " 3 " judgments and a larger standard deviation in the averaged midpoints gained from the probit analysis. This may explain the nonsignificant probability level of the difference between the judgments made for the two stimulus patterns $[F(1,33)=2.185, p=.1488]$, even though the midpoints were very close to those found in Experiment 1.

We had been uncertain as to whether the different tasks would affect the perceptual grouping since the subjects were essentially being asked to judge the same two attributes, pitch and tempo. It appears that the subjects had little trouble identifying the tempo of a stream and then making a judgment about its pitch as in Experiment 1 , but doing the same thing in the reverse order (as in Experiment 2) presented problems. The mean responses were similar for both tasks, although there were more " 3 " judgments made in the second experiment. It appears that the task did influence the ease with which the subjects could identify the perceptual attributes of the streams, but that it did not influence the allocation of components to streams.

The "transition points," at around $1180 \mathrm{~Hz}$ for the synchronous pattern and $1280 \mathrm{~Hz}$ for the sequential pattern, were unexpectedly low. For both patterns, $\mathrm{V}$ had to be substantially closer to the lower tone than either the lin- 
ear or logarithmic midpoints ( 1451 and $1354 \mathrm{~Hz}$, respectively) in order to avoid being grouped with the upper tone. If the simple proximity of tones in cochlear location were a sufficient determinant of grouping (a too-simple expectation, really, in view of the nonlinear and asymmetrical mechanisms of cochlear filtering), the transitions might have agreed more closely with the midpoint on a cochlear map. However, the midpoint between $979 \mathrm{~Hz}$ $(13.677 \mathrm{~mm})$ and $1973 \mathrm{~Hz}(18.525 \mathrm{~mm})$ given by Greenwood's $(1961,1990)$ frequency-place map is $1364 \mathrm{~Hz}$ $(16.102 \mathrm{~mm})$, which is the same midpoint determined by measuring distance in critical bandwidth or ERB units (Greenwood, 1991; Moore \& Glasberg, 1983). The Mel scale (Stevens \& Volkmann, 1940), which suggests a similar midpoint of around $1390 \mathrm{~Hz}$, was found through an equisection experiment done for Stevens in 1956 (Greenwood, 1997) to have apparently been biased. However, the equal pitch distances obtained in the 1956 experiment on bias correspond closely to equal distances on the cochlear map, thus indicating the same midpoint as on the map - that is, the midpoint found not to describe our grouping transition point.

But it is not too surprising (even if not yet predictable) that the auditory system, in determining the relative "independence" of the lower and upper tones when the intermediate tone is grouped with one or the other, depends on factors in addition to their spacing along the cochlear map or their pitch differences. It is also evident, attesting to the existence of such factors, that grouping is constrained by the different mechanisms and interactions that must occur in simultaneous, as opposed to sequential, presentations of the upper and lower tones - which is more a causal view of this dependence than calling it, more generally, a dependence on the temporal arrangements of the tones without explicitly citing their overlap. The different midpoints found for the two patterns thus demonstrate that the streaming effect is not due solely to the proximity of the frequency components but depends also on differences in the operative mechanisms and interactions when the tones are simultaneous rather than sequential.

Since the lower tone has been shown in this experiment to be more dominant in resisting grouping with another tone in the phenomenon of streaming, it should be interesting and productive to seek other evidence of a dominant role for the lower frequency component in other streaming paradigms that may be devised and in experiments devoted to other pitch phenomena.

\section{REFERENCES}

Bregman, A. S. (1978). Auditory streaming: Competition among alternative organizations. Perception \& Psychophysics, 23, 391-398.

Bregman, A. S. (1990). Auditory scene analysis. Cambridge, MA: MIT Press.

Bregman, A. S., \& Pinker, S. (1978). Auditory streaming and the building of timbre. Canadian Journal of Psychology, 32, 19-31.
Finney, D. J. (1971). Probit analysis. Cambridge: Cambridge University Press.

Fletcher, H. (1940). Auditory patterns, Review of Modern Physics, 12, 47-65.

Glasberg, B. R., \& Moore, B. C. J. (1990). Derivation of auditory filter shapes from notched noise data. Hearing Research, 47, 103-138.

Greenwood, D. D. (1961). Critical bandwidth and frequency coordinates of the basilar membrane. Journal of the Acoustical Society of America, 33, 1344-1356.

GreENwoOD, D. D. (1990). A cochlear frequency-position function for several species-29 years later. Journal of the Acoustical Society of America, 87, 2592-2605.

GrEenwooD, D. D. (1991). Critical bandwidth and consonance in relation to cochlear frequency-position coordinates. Hearing Research, 54, 164-208.

Greenwoon, D. D. (1997). The Mel Scale's disqualifying bias and a consistency of pitch-difference equisections in 1956 with equal cochlear distances and equal frequency ratios. Hearing Research, 103, 199-248.

HENKE, W. L. (1990). MITSYN: A synergistic family of high-level languages for time signal processing (Version 8.I). (Available from William L. Henke, 133 Bright Road, Belmont, MA 02178)

HoutGaST, T. ( 1977). Auditory filter characteristics derived from directmasking data and pulsation threshold data with a rippled-noise masker. Journal of the Acoustical Society of America, 62, 409-415.

MAYer, A. M. (1894). Researches in acoustics-IX. Philosophical Magazine-5th Series, 37, 259-288.

Miller, G. A., \& Heise, G. A. (1950). The trill threshold. Journal of the Acoustical Society of America, 22, 637-638.

Moore, B. C. J., \& Glasberg, B. R. (1983). Suggested formulae for calculating auditory-filter bandwidths and excitation patterns. Journal of the Acoustical Society of America, 74, 750-753.

Moore, B. C. J., Peters, R. W., \& GlasberG, B. R. (1990). Auditory filter shapes at low center frequencies. Journal of the Acoustical Society of America, 88, 132-140.

Patterson, R. D. (1976). Auditory filter shapes derived in simultaneous and forward masking. Journal of the Acoustical Society of America, 70, 1003-1014.

Plomp, R., \& SteEneken, H. J. (1968). Interference between two simple tones. Journal of the Acoustical Society of America, 43, 883-884.

SCHARF, B. (1975). Audition. In B. Scharf (Ed.), Experimental sensory psychology (pp. 112-149). Glenview, IL: Scott, Foresman.

Shailer, M. J., \& Moore, B. C. J. (1983). Gap detection as a function of frequency, bandwidth and level. Journal of the Acoustical Society of America, 74, 467-473.

Shailer, M. J., Moore, B. C. J., Glasberg, B. R., Watson, N., \& HarRIS. S. (1990). Auditory filter shaped at 8 and $10 \mathrm{kHz}$. Journal of the Acoustical Society of America, 88, 141-148.

Stevens, S. S., \& VolkmanN, J. (1940). The relation of pitch to frequency: A revised scale. American Journal of Psychology, 53, 329-353.

VAN NoORden, L. P. A. S. (1975). Temporal coherence in the perception of tone sequences. Unpublished doctoral dissertation, Eindhoven University of Technology, The Netherlands.

WEBER, D. L. (1977). Growth of masking and the auditory filter. Journal of the Acoustical Society of America, 62, 424-429.

ZWICKER, E. (1970). Masking and psychological excitation as consequences of the ear's frequency analysis. In R. Plomp \& G. F. Smoorenburg (Eds.), Frequency analysis and periodicity detection in hearing (pp. 278-287). Leiden: Sijthoff

ZWICKER, E.. FlotTorP, G., \& STEvens, S. S. (1957). Critical bandwidth in loudness summation. Journal of the Acoustical Society of America, 29, 548-557.

(Manuscript received July 28, 1997; revision accepted for publication September 28, 1998.) 